\title{
Non-linear resonance model for black hole and neutron star QPOs: observation and theory
}

\author{
Gabriel Török $^{1,2}$, Marek A. Abramowicz ${ }^{1,2,3}$, Włodek Kluźniak ${ }^{2,4}$, \\ Zdeněk Stuchlík ${ }^{1}$, Eva Šrámková ${ }^{1,2}$ \\ ${ }^{1}$ Institute of Physics, Faculty of Philosophy and Science, Silesian University in Opava, \\ Bezručovo nám. 13, CZ-746 01 Opava \\ ${ }^{2}$ Copernicus Astronomical Centre, Polish Academy of Sciences, Bartycka 18, PL-00 716 \\ Warsaw, Poland Department of Physics \\ ${ }^{3}$ Göteborg University, S-412 96 Göteborg, Sweden \\ ${ }^{4}$ Insitute of Astronomy, Zielona Góra University, Lubuska 2, 65-265 Zielona Góra, Poland \\ E-mail: terek@volny.cz, marek@fy.chalmers, wlodek@camk.edu.pl, \\ zdenek.stuchlik@fpf.slu.cz, sram_eva@centrum.cz
}

Kilohertz Quasi-Periodic Oscillations (QPOs) have been detected in many accreting X-ray binaries. It has been suggested that the $\mathrm{kHz}$ QPO frequencies observed in the modulation of the $\mathrm{X}$-ray flux reflect a non-linear resonance between two modes of accreting disk oscillations. A 3:2 resonant ratio of frequencies has been clearly recognized in the black-hole QPO data supporting the non-linear resonance hypothesis. It is often discussed whether the same 3:2 ratio appears in case of the neutron star QPOs as well. For an individual neutron star source, the relation between the observed lower and upper $\left(v_{L}\right.$ and $\left.v_{U}\right) \mathrm{kHz}$ QPO frequency is well approximated by a linear relation with the slope $A$ and the shift $B$, i.e., $v_{U}=A v_{L}+B$. It was recognized only recently that for a group of twelve neutron star sources the value of the individual coefficients $A, B$ is anticorrelated, obeying the condition $A=3 / 2-0.0016 B$. It was also shown that such an anticorrelation is predicted by the theory of weakly coupled nonlinear oscillators, and one can argue that it indeed pose the evidence for a 3:2 resonant ratio. Here, stressing the latest progress, we discuss some of the links between the QPO observation and the resonance model, and summarize the appropriate references.

Except the slightly updated list of references, the content of next four pages fully correspond to the AO poster we exhibited at the VI Microquasar Workshop. The poster itself is available at the http://www.physics.cz/research (section Conferences).

VI Microquasar Workshop: Microquasars and Beyond

September 18-22, 2006

Como, Italy 


\section{Observation}

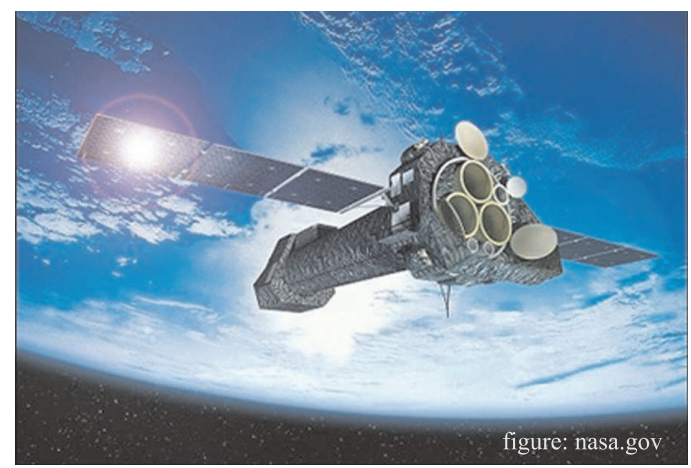

Many black hole (microquasar) and neutron star sources in low mass $X$-ray binaries show QPOs (quasi periodic oscillations) in their observed $X$-ray fluxes, i.e., peaks in the Fourier variability power density spectra (PDS). QPOs are also observed in the Galaxy centre black hole $\mathrm{Sgr}^{*}$ and in a few galactic nuclei. Frequencies of some QPOs are in the $\mathrm{kHz}$ range which corresponds to orbital frequencies close to the central compact object. A special class of high frequency QPOs consists of twin peak $Q P O$ s, i.e., those which show a pair of peaks in their PDS - two frequencies $\nu_{L}, \nu_{U}$. These twin peak QPOs were recently reported also in the peculiar microquasar (neutron star candidate) Circinus $X-1$.
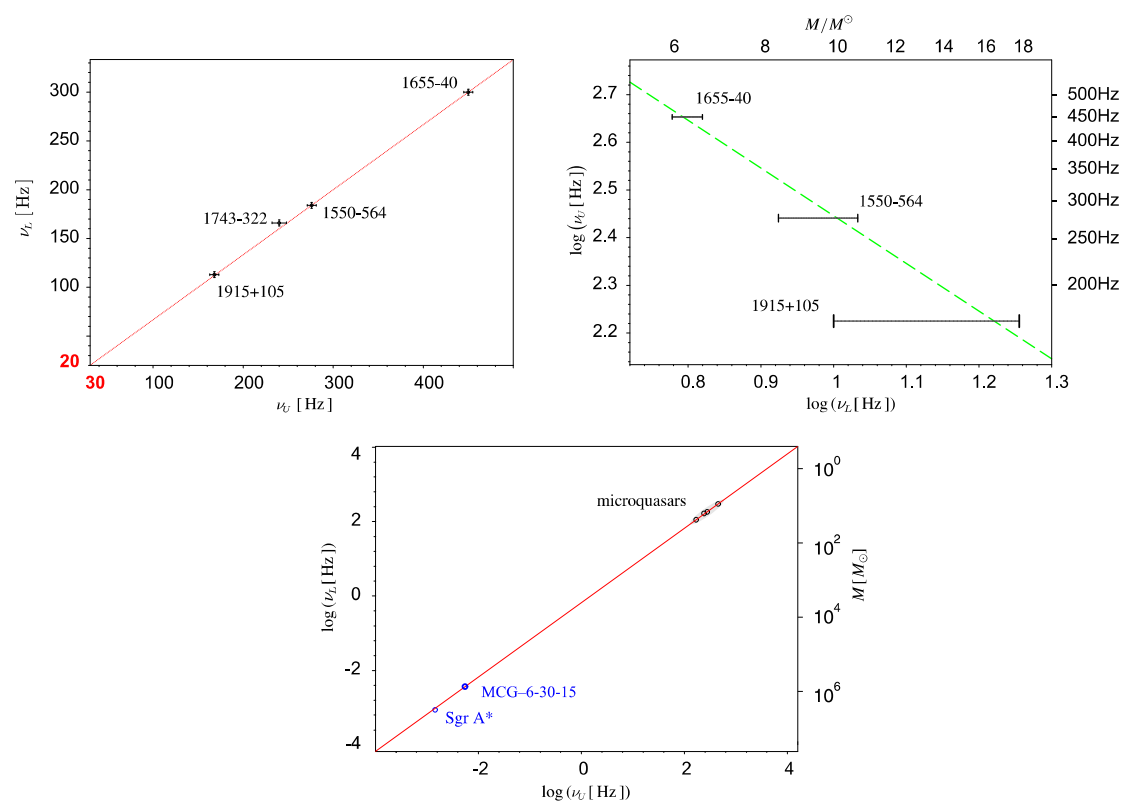

Figure 1: Left: The microquasar twin peak kHz QPOs frequencies in a 3:2 ratio. Right: the inverse mass scaling for microquasars found by [10]. Bottom: 3:2 ratio appears also in the Galaxtic centre black hole and two extragalactic sources (MCG 6-30-15, NGC 40-51 [11]). The $1 / M$ scaling can be (at least roughly) extrapolated in the same way as well.

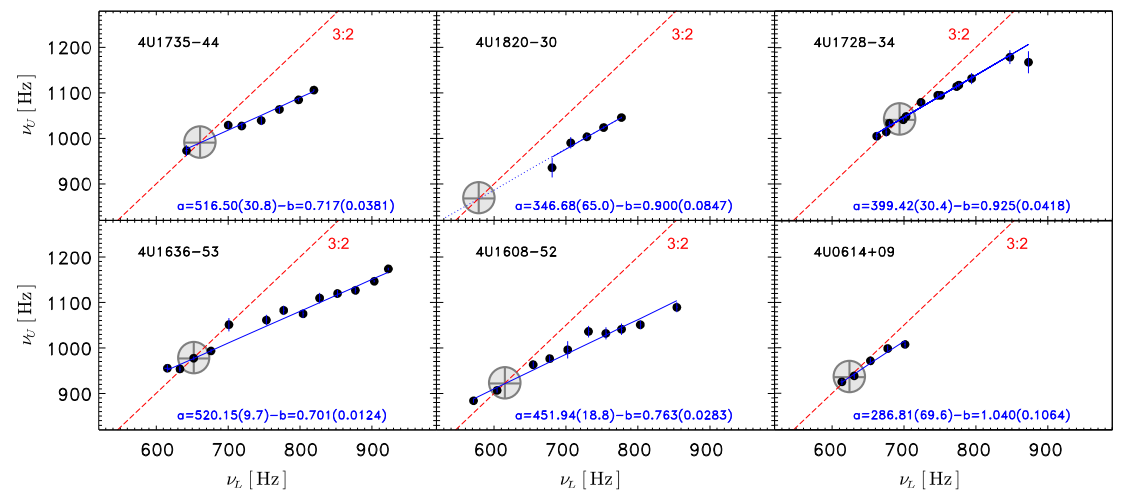

Figure 2: For each of the six atoll sources examined in $[9,15]$, the linear correlation (best fit denoted by blue line) well fits the upper vs. the lower QPO frequency. The cross-hair marks denote the zero points of the s-parameter (see Section 2). 

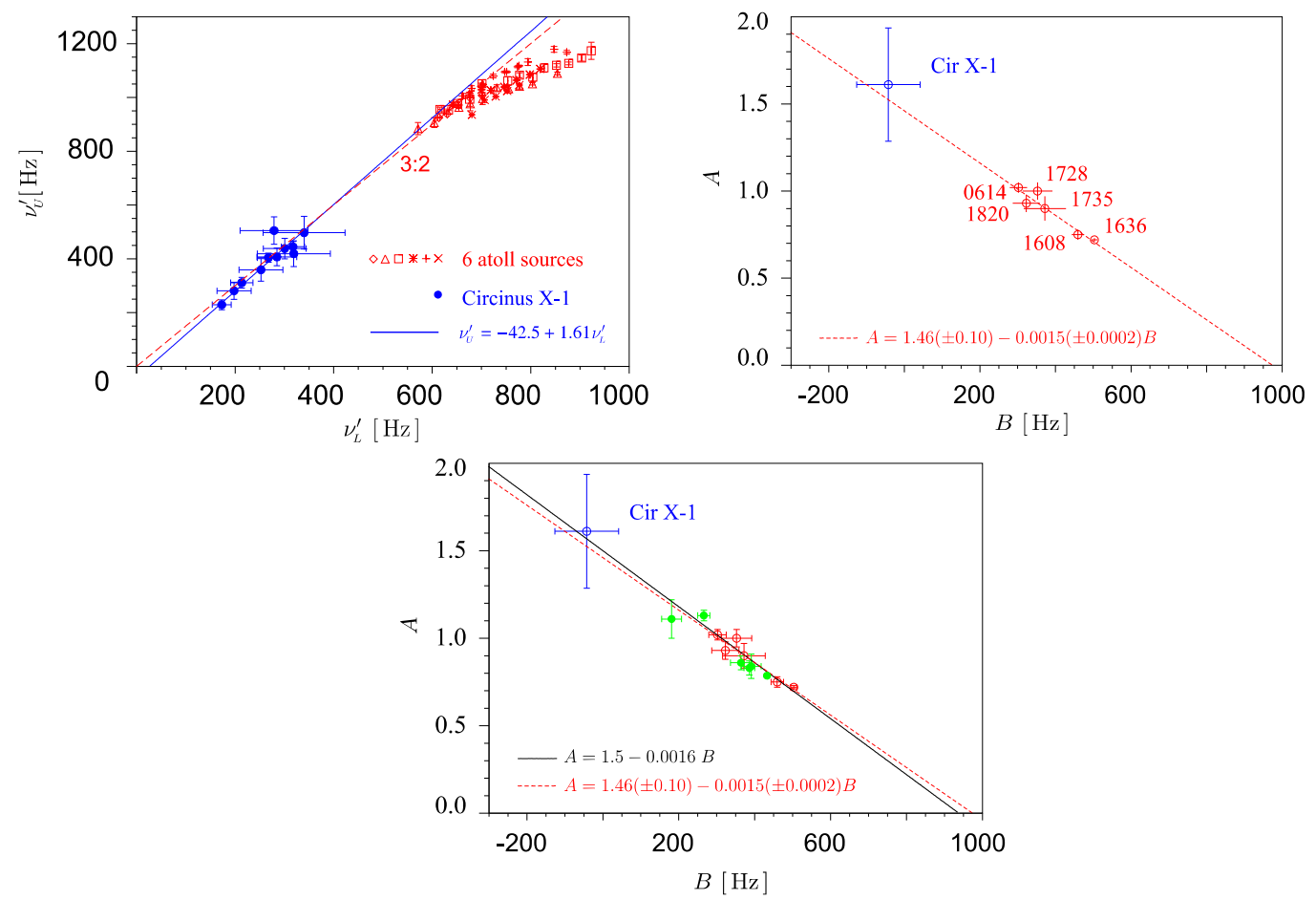

Figure 3: The slope-shift anticorrelation $[9,15]$. Left: Frequencies $v_{L}^{\prime}, v_{U}^{\prime}$ supposed to be in $3: 2$ resonance identified in six atoll sources (Figure 2) directly with the observed frequencies $v_{L}^{\prime}=v_{L}, v_{U}^{\prime}=v_{U}$, and as $v_{L}^{\prime}=v_{U}^{\prime}-v_{L}, v_{U}=v_{U}^{\prime}$ in the case of Cir X-1 (data from [13]). Right: The dashed line represents the best fit of Bursa line coefficients for six atoll sources (Figure 2). The Cir X-1 coefficients corresponding to the combinational frequency hypothesis $v_{L}^{\prime}=v_{L}, v_{U}^{\prime}=v_{U}$ are denoted [14]. Bottom: $[9,15]$ have also shown that the other six sources (the milisecond pulsar, one atoll, and four $\mathrm{Z}$ sources; denoted here by green circles) follow the same trend. Remarkably, all the thirteen sources including Cir X-1 are within one sigma level consistent with relation $A=1.5-0.0016 B$.
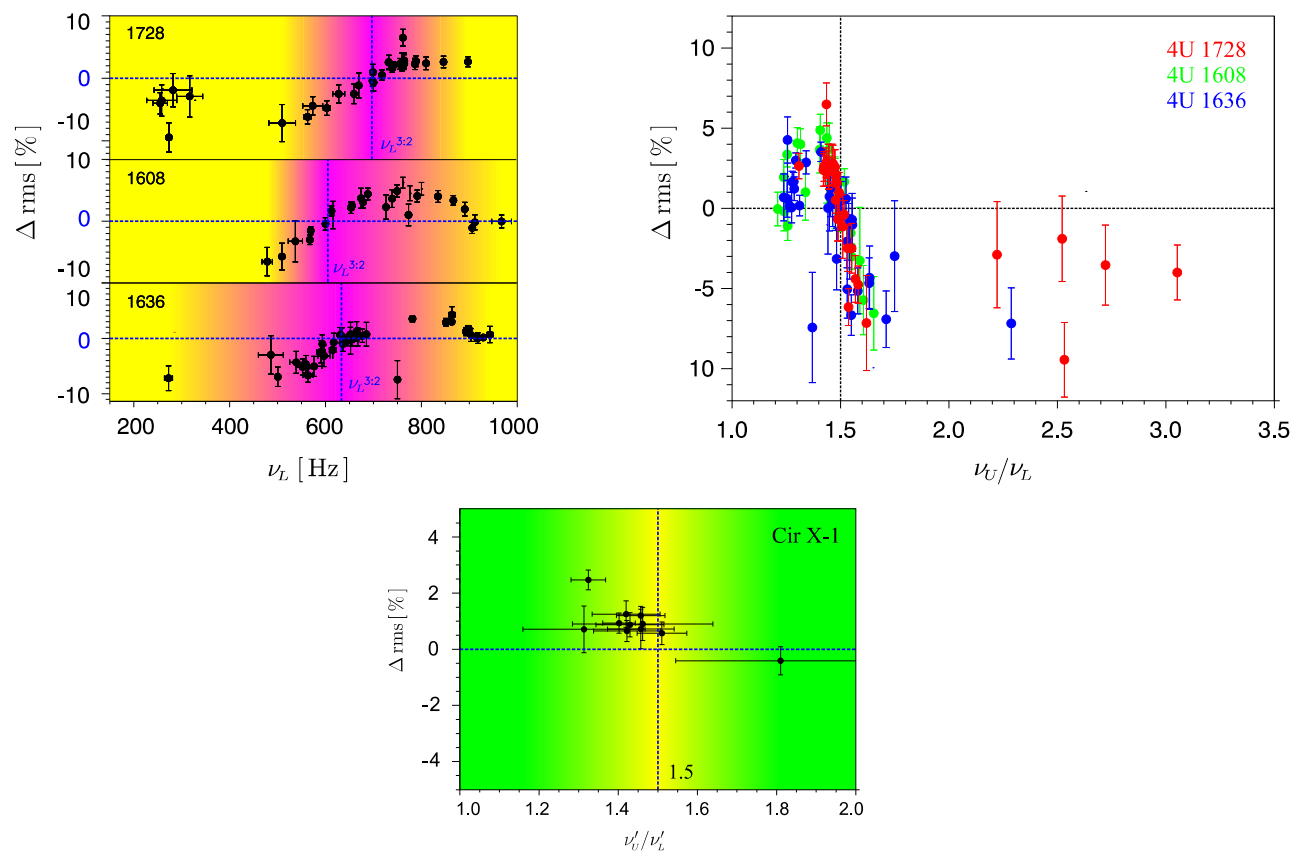

Figure 4: The energy switch effect $[12,16]$. Left: The difference of the rms amplitudes vs. lower QPO frequency for three atoll sources $4 \mathrm{U} 1728-34,4 \mathrm{U} 1608-52$, and $4 \mathrm{U} 1636-53$. The quantity $\Delta \operatorname{rms}\left(v_{L}\right) \equiv A_{r m s}^{\mathrm{L}}\left(v_{L}\right)-A_{r m s}^{\mathrm{U}}\left(v_{L}\right)$ changes its sign across the 3:2 resonance line. Up to the 'resonance point' the amplitudes are larger in the case of the upper frequency, equal when 'Bursa line' pass 3:2 ratio and above this, the lower QPO has the stronger amplitude. Right: The rms amplitude difference in the case of Cir X-1 [14], data from [13]. 


\section{Theory}

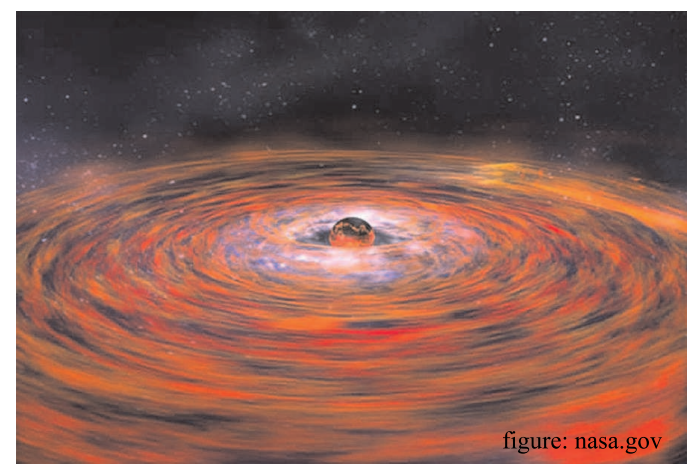

It has been suggested by Kluźniak \& Abramowicz [1] that the highest quasi periodic oscillation frequencies observed in the modulation of the X-ray flux reflect a non-linear resonance between two modes of accretion disk oscillations. This hypothesis implies certain very general predictions, several of which have been born out by observations. Some of these follow from the properties of non-linear oscillators, while the others are specific to oscillations of fluid in strong gravity.

\subsection{Orbital resonance model}

The orbital resonance model [2] demonstrates that fluid accretion flows admit two linear quasiincompressible modes of oscillations, vertical and radial, with corresponding eigenfrequencies equal to vertical and radial epicyclic frequencies for free particles [3, 4]. In the model of slender torus, the general properties of these modes can be shown: the vertical mode corresponds to a periodic displacement in which the whole torus moves as a rigid body up and down the equatorial plane and each fluid element has a vertical velocity that periodically changes in time. The frequency of the vertical mode is equal to the vertical epicyclic frequency that a ficticious free particle orbiting at the circle of maximum pressure in the torus equilibrium position would have. Behaviour of the radial mode is similar to the vertical one, and in the linear regime these two modes are formally uncoupled. In the case of more realistic description that includes non-linear effects given by pressure and dissipation, these effects couple the two epicyclic modes that may result in a resonance [2]. As shown by [5], in the Kerr geometry, the strongest resonance between the epicyclic frequencies can occur for $v_{\theta}: v_{r}=3: 2$.

\subsubsection{Description of modes}

It was recently found that pressure and other non-geodesic effects have stronger influence on the frequencies of some modes than previously thought [6]. The work [6] focusses on properties of vertical and radial epicyclic modes comparing the case of slender and non-slender torus transforming one case to another one through formall parameter $\beta$ using the Paczynski-Wiita potencial. In this Pseudo-Newtonian result, the frequencies of oscillations are slightly changing with $\beta$ which causes a drift of the frequency ratio with $\beta$, and resonant frequencies increase.

This phenomenon introduces uncertainty in the direct comparison of QPO frequencies with the model - the effect could change the rough estimate of neutron star masses given by identification of the eigenfrequencies derived from the slope-shift anticorrelation with 3:2 epicyclic frequencies for free particles. A similar effect in the Kerr metric can also affect the black hole spin parameter estimated for a given QPO frequency [7]. 

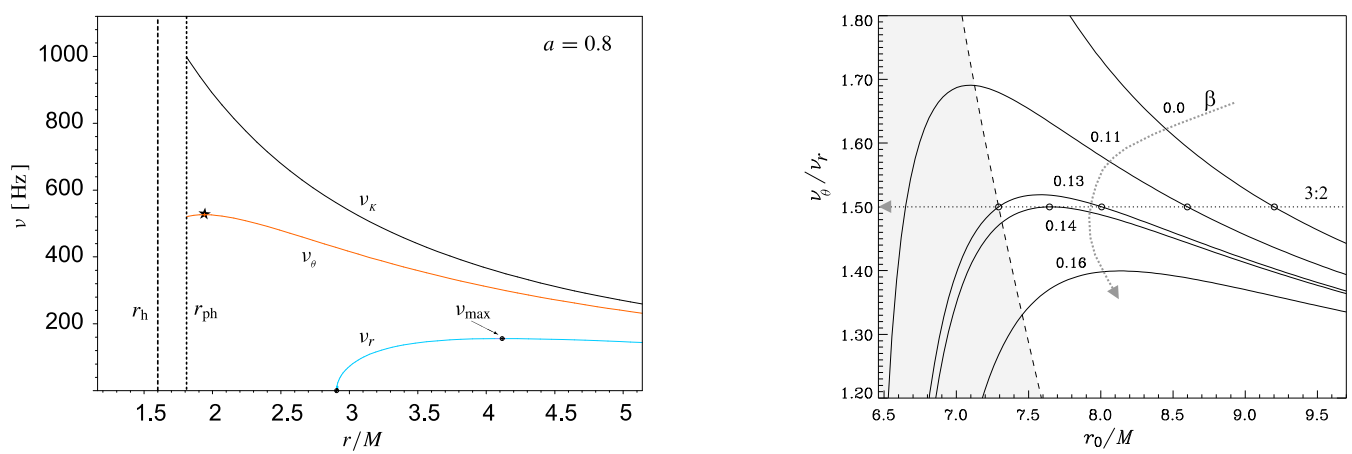

Figure 5: Left: Behaviour of epicyclic frequencies typical for Kerr black holes. In the gravitational field of a central object, test particle on a circular orbit starts oscillating after a small perturbation. Frequencies of this oscillations (radial $v_{\mathrm{r}}$ and vertical $v_{\theta}$ ) are fundamentally different in Newton's and Einstein's gravity. In Newtonian physics the epicyclic frequencies are equal to the Keplerian frequency of the circular orbit and the resulting trajectory is an ellipse, while in Einstein's theory they differ and the trajectory is not closed. Recall also that compared to the Newtonian case, all the three frequencies scale inversely with the mass generating the gravitational field, and for the Kerr black holes depend also on the black hole spin. Right: Position of the center of a torus fulfilling the $3: 2$ resonance condition, as a function of the parameter $\beta$ characterizing thickness of the torus [6].

\subsection{Few general properties of non-linear oscillators}

\subsubsection{Resonance conditions}

Parametric resonance between two oscillation modes $\omega_{\theta}, \omega_{r}$ is described by the Mathieu equation [8] $\delta \ddot{\theta}+\omega_{\theta}^{2}\left[1+h \cos \left(\omega_{r} t\right)\right] \delta \theta=0$. Such a parametric or an internal resonance [5] can occur if

$$
\frac{\omega_{r}}{\omega_{\theta}}=\frac{v_{r}}{v_{\theta}}=\frac{2}{n}, \quad n=1,2,3 \ldots
$$

and is strongest for the smallest possible value of $n$. Forced resonances can arise if

$$
\delta \ddot{\theta}+\omega_{\theta}^{2} \delta \theta+\mathscr{O}^{2}(\delta \theta)=h(r) \cos \left(\omega_{r} t\right), \frac{\omega_{\theta}}{\omega_{r}}=\frac{p}{q}, p, q=1,2,3 \ldots
$$

In all these cases, resonance occurs if the frequencies are in the ratio of small natural numbers.

\subsubsection{The slope-shift anticorrelation}

For a non-linear oscillator, the observed frequency of oscillations $v$ differs from the eigenfrequency $v^{\circ}$ by a correction proportional to the square of a small, dimensionless amplitude $\alpha$ [8]. System having two oscillation modes [5]:

$$
\begin{gathered}
v_{U}=v_{U}^{\circ}+v_{U}^{\circ}\left(C_{U U} \alpha_{U}^{2}+C_{U L} \alpha_{L}^{2}\right)+\mathscr{O}^{3}(\alpha), \\
v_{L}=v_{L}^{\circ}+v_{L}^{\circ}\left(C_{U} \alpha_{U}^{2}+C_{L} \alpha_{L}^{2}\right)+\mathscr{O}^{3}(\alpha),
\end{gathered}
$$

If the two amplitudes are arbitrarily correlated the actual frequencies of the oscillations are correlated as well: $v_{U}=v_{U}^{\circ}+F(s), \quad v_{L}=v_{L}^{\circ}+G(s)$. For a weak non-linearity and coupling $F(s)=$ $F_{0} \cdot s+\mathscr{O}\left(s^{2}\right), G(s)=G_{0} \cdot s+\mathscr{O}\left(s^{2}\right)\left[s=0 \Leftrightarrow v_{U}=v_{U}^{\circ}\right.$ and $\left.v_{L}=v_{L}^{\circ}\right]$

$$
v_{U}=A v_{L}+B ; \quad A=X, \quad B=v_{U}^{\circ}-X v_{L}^{\circ}, \quad X \equiv\left(\frac{F_{0}}{G_{0}}\right) .
$$

Note that $A$ and $B$ do not depend on $s$ and equation (2.4) describes a straight line in $v-v$ plane (which we call the Bursa line). 
Let us call source such two weakly coupled oscillators. For group of sources having the same eigenfrequencies the physical details projected to the functions $F, G$ differ. However, one can eliminate the quantity $X$ and thus get universal relation between individual coefficients $A^{k}, B^{k}$ :

$$
A^{k}=A^{0}-\gamma B^{k} ; \quad \gamma=\frac{1}{v_{L}^{\circ}}\left[1+\mathscr{O}\left(\alpha^{2}\right)\right], A^{0}=\frac{v_{U}^{\circ}}{v_{L}^{\circ}}+\mathscr{O}\left(\alpha^{2}\right) .
$$

Obviously, the slope A and shift B of individual Bursa lines are anticorrelated [9] as all the lines cross at single point $\left[v_{L}^{\circ}, v_{U}^{\circ}\right]$. For a set having the same eigenfrequency ratio with individual scatter $\varsigma^{\mathrm{k}}$ around $\left[v_{U}^{\circ}, v_{L}^{\circ}\right]$ the anticorrelation reads $A^{\mathrm{k}}=A^{0}-\frac{1}{\varsigma^{\mathrm{k}} v_{L}^{\circ}} B^{\mathrm{k}}$.

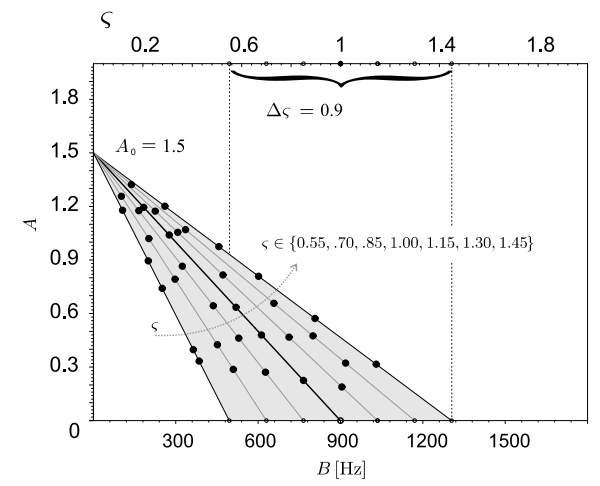

Figure 6: The anti-correlation triangle for 35 hypothetical sources all having the eigenfrequency ratio equal to 1.5 .

\section{References}

[1] W. Kluźniak, M. A. Abramowicz, The physics of kHz QPOs—strong gravity's coupled anharmonic oscillators, Acta Phys. Polon., 2001, B32, 3605, [astro-ph/0105057].

[2] W. Kluźniak, M. A. Abramowicz, Parametric epicyclic resonance in black hole disks: QPOs in micro-quasars, 2002 [astro-ph/0203314]

[3] A. N. Aliev, D. V. Galtsov, Radiation from relativistic particles in nongeodesic motion in a strong gravitational field, General Relativity and Gravitation, 1981, 13, p. 899

[4] M. Nowak, D. Lehr, Stable oscillations of black hole accretion discs, in Theory of Black Hole Accretion Disks, eds. M.A. Abramowicz,G. Björnsson, J. E. Pringle, Cambridge, 1998)

[5] J. Horák, General aspects of nonlinear resonance 3:2 in QPO context, in Proceedings of RAGtime 5, eds. S. Hledík, Z. Stuchlík, Silesian University at Opava, 2004

[6] O. Blaes, E. Šramková, M. A. Abramowicz, W. Kluźniak, U. Torkelsson, et al., Epicyclic Oscillations of Fluid Bodies: Newtonian Non-Slender Torus, ApJ, 2006, submitted.

[7] G. Török, M. A. Abramowicz, W. Kluźniak, Z. Stuchlík, The orbital resonance model for twin peak kHz quasi periodic oscillations in microquasars, A\&A, 2005, 436, 1, p. $1-8$, see also recent astro-ph/0610497

[8] L. D. Landau, E. M. Lifshitz, Mechanics, Oxford, 1976

[9] M. A. Abramowicz, D. Barret, M. Bursa, J. Horák, W. Kluźniak, P. Rebusco, and G. Török, A note on the slope-shift anticorrelation in the neutron star kHz QPOs data, in Proceedings of RAGtime 6/7, eds. S. Hledík, Z. Stuchlík, Opava, 2005, see also

- M.A. Abramowicz, T. Bulik, M. Bursa, W. Kluźniak, Evidence for a 2:3 resonance in Sco X-I kHz QPOs, A\&A, 404, 2003

- T. Belloni, M. Méndez, J. Homan, The distribution of $k H z$ QPO frequencies in bright low mass $X$-ray binaries, A\&A, 437, 2005

[10] J. E. McClintock, R. A. Remillard, Black hole binaries, in Compact Stellar X-Ray Sources, eds. W. H. G. Lewin, M. van der Klis, Cambridge, 2004 [astro-ph/0306213]

[11] P. Lachowicz, B. Czerny, M. A. Abramowicz, Wavelet analysis of MCG-6-30-15 and NGC 4051: a possible discovery of QPOs in 2:1 and 3:2 resonance, 2006 [astro-ph/0607594]

[12] Török, Barret, in preparation

[13] S. Boutloukos, M. van der Klis, D. Altamirano, M. Klein-Wolt, R. Wijnands, P.G. Jonker, R.P. Fender, Discovery of twin kHz QPOs in the peculiar X-ray binary Circinus $X-1$, submitted to ApJ, 2006, [astro-ph/0608089]
G.

[14] G. Török, Z. Stuchlík, M. A. Abramowicz, W. Kluźniak, in preparation

[15] M. A. Abramowicz, D. Barret, M. Bursa, J. Horák, W. Kluźniak, P. Rebusco, G. Török, The slope-intercept anti-correlation in neutron-star kHz QPOs, MNRAS, 2006, submitted

[16] Török, Abramowicz, Kluźniak, Stuchlík, A non-linear resonance model for the black hole and neutron star QPOs: theory supported by observations, proc. of Albert Einstein Conf. Paris 2005, 2006, in print [astro-ph/0603847]

[17] www.physics.cz/research \& http://cta.cas.cz]

Supported by the grants CZ MSM 4781305903 (MA,ZS,GT), 1P03D 00530 (WK), and LC06014 (ES).
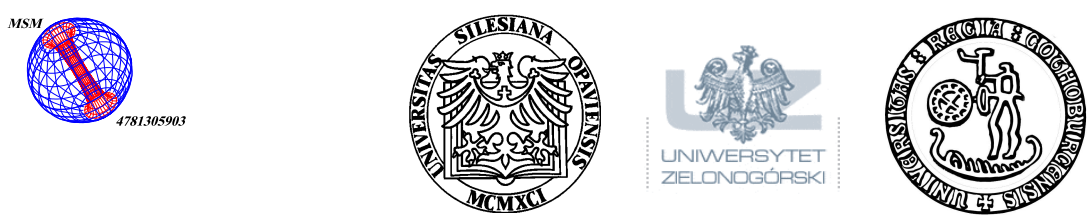